\title{
GCU
}

Glasgow Caledonian

University

University for the Common Good

\section{Analysis of PAPR in optical OFDM systems with grouped LEDs}

Ogunkoya, Funmilayo B.; Popoola, Wasiu O.; Sinanovic, Sinan

Published in:

Optik: International Journal for Light and Electron Optics

DOI:

10.1016/j.ijleo.2017.08.001

Publication date:

2017

Document Version

Author accepted manuscript

Link to publication in ResearchOnline

Citation for published version (Harvard):

Ogunkoya, FB, Popoola, WO \& Sinanovic, S 2017, 'Analysis of PAPR in optical OFDM systems with grouped LEDs', Optik: International Journal for Light and Electron Optics, vol. 151, pp. 48-54.

https://doi.org/10.1016/j.ijleo.2017.08.001

\section{General rights}

Copyright and moral rights for the publications made accessible in the public portal are retained by the authors and/or other copyright owners and it is a condition of accessing publications that users recognise and abide by the legal requirements associated with these rights.

Take down policy

If you believe that this document breaches copyright please view our takedown policy at https://edshare.gcu.ac.uk/id/eprint/5179 for details of how to contact us. 


\title{
Analysis of PAPR in Optical OFDM Systems with Grouped LEDs
}

*Funmilayo B. Offiong, Wasiu O. Popoola, Sinan Sinanović,

\begin{abstract}
The need for multiple transmit chains poses a major challenge in optical orthogonal frequency division multiplexing (OFDM) system utilising grouped light emitting diodes (LEDs) for the purpose of peak-to-average power ratio (PAPR) reduction. Thus, recent study propose the use of pilot-assisted (PA) electrical PAPR reduction technique to further reduce PAPR and limit the number of transmit chains in the system. This paper presents the application of order statistics to derive the analytical solutions of PAPR distributions in the PA optical OFDM system utilising grouped LEDs. It is shown that the results obtained via analysis are in good agreement with that of computer simulations. Keywords: Optical OFDM; grouped LEDs; PAPR reduction analysis; pilot symbol; order statistics.
\end{abstract}

\section{Introduction}

Wireless data communication traffic is witnessing exponential growth primarily due to increase in the number of subscribers and smart portable devices per subscriber [1]. This rate of growth implies challenges for existing radio frequency (RF) communication system to meet high traffic demands. A potential solution to complement existing RF is the use of optical wireless communications (OWC) [2]. The prospect of OWC using visible part of the spectrum, called visible light communication (VLC), to meet high data rate demands has been

\footnotetext{
* Corresponding author

Email address: fboffiong@ieee.org ()
} 
demonstrated in [3] using orthogonal frequency division multiplexing (OFDM) transmission technique. Spectrally efficient optical OFDM (O-OFDM) will continue to gain popularity in VLC due to its ability to maximise the use of limited modulated bandwidth of light emitting diodes (LEDs) 4] ]. However, the problem of high peak-to-average power ratio (PAPR) must be mitigated in O-OFDM in order to fully exploit the huge spectrum resources available for use in VLC.

The dynamic range of LEDs is limited [5]. This limitation makes it challenging to modulate the full amplitude swings of signal with high peaks on LED intensity without clipping. As a result, the clipping causes signal distortion which degrades the error performance of the signal. Significant research effort is being directed towards the PAPR reduction technique in O-OFDM. Among the techniques is the use of post-coding scheme based on discrete cosine transform (DCT) implemented after the inverse fast Fourier transform (IFFT) operation to reduce the PAPR of O-OFDM signals [6]. Embedding pilot symbol in the original O-OFDM signal in frequency domain for the purpose of PAPR reduction is proposed in [4]. Another method that generates series of Toeplitz matrix using the time domain signal out of which the O-OFDM signal with the lowest PAPR is selected for transmission is presented in [7]. Authors in [8, 9, 10] proposed spatial domain O-OFDM utilising grouped LEDs as a means of transmitting O-OFDM signal with reduced peaks.

It is a common practice to illuminate an indoor environment with panels of luminaire having multiple LEDs for sufficient and uniform illumination [11]. Thus, the spatial domain O-OFDM approach takes advantage of the multiple LEDs to mitigate some of the existing problems of O-OFDM in VLC [8, 9 , 10]. O-OFDM signal achieved via spatial summing involves partitioning the subcarriers for transmission over different groups of LEDs. However, the number of transmit chains increases as the number of LED groups increases. Thus, study reported in [12, 13] proposed the use of pilot-assisted (PA) electrical PAPR reduction technique to further reduce PAPR and limit the number of transmit chains in the system. While PA O-OFDM system using grouped LEDs is investigated in 12, 13], a quantitative analysis of its PAPR distributions has 
never been reported in literature. The theoretical work presented in [14] is based on PAPR distributions of the PA O-OFDM signal

In this work, we extend the study of the PA technique in spatial domain O-OFDM utilising grouped LEDs to theoretically characterise the PAPR distributions and PAPR reduction gain using order statistics. Application of order statistics as analytical tool has been reported in varieties of fields ranging from life sciences to radio frequency (RF) wireless communications [15]. Areas where order statistics have been applied in RF wireless systems include transmission and reception techniques involving diversity combining, multiuser scheduling and channel adaptation in multiple-input-multiple-output (MIMO) and OFDM [15]. In this paper, we apply order statistics to quantify the PAPR distributions of the PA O-OFDM utilising grouped LEDs. Closed-form result of the PAPR distributions of the technique is presented using the complementary cumulative distribution function (CCDF), which gives the probability of a PAPR value exceeding a certain threshold level, $y$. PAPR is an important metric of interest employed in an OFDM system for estimating the dynamic range of the digitalto-analog converter (DAC) 16]. Thus, theoretical framework of the electrical PAPR distributions in PA O-OFDM will serve as a guideline for implementation of the technique in multiple LEDs systems.

The analytical framework for evaluating the PAPR distributions and PAPR reduction gain using order statistics is validated with computer simulation. Moreover, this work will include the comparison of the PA technique in OOFDM using multiple LEDs analytical solutions with the basic O-OFDM counterpart where PA technique is not implemented.

The rest of this paper is organized as follows: Section 2 provides a review of the PA O-OFDM system utilising grouped LEDs. Section 3 presents the analysis of the system. Results comparing performance of the PA O-OFDM system utilising grouped LEDs with its basic O-OFDM counterpart are presented in 4 Finally, Section 5 concludes the paper. 

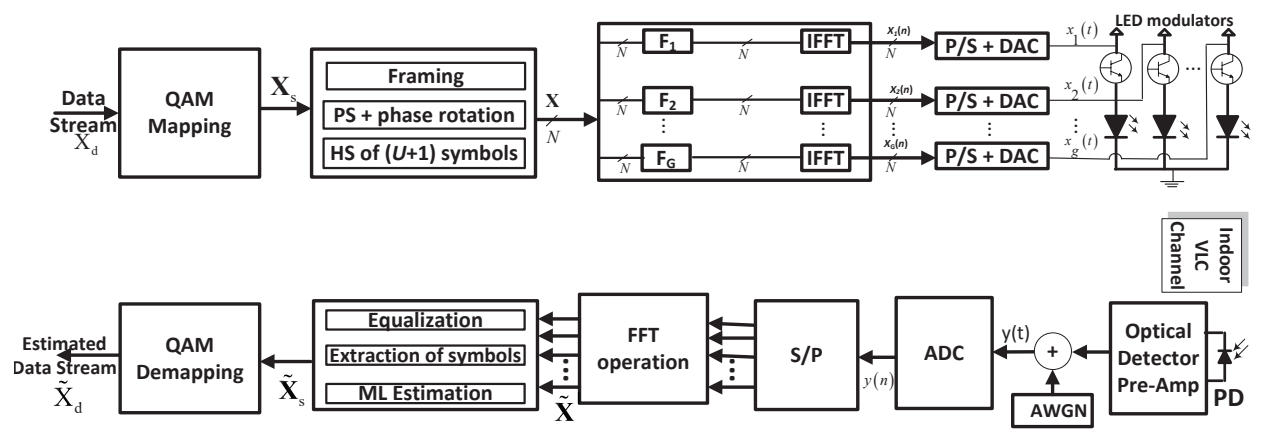

Figure 1: Block diagram illustrating the architecture of the pilot-assisted O-OFDM system with multiple LED groups. PS: pilot symbol, HS: Hermitian symmetry, P/S: Parallel-to-serial, S/P:serial-to-parallel, ADC: analog-to-digital converter, ML: Maximum likelihood

\section{Review of the Pilot-assisted O-OFDM System with Grouped LEDs}

Figure 1 shows the schematic block diagram of the PA O-OFDM utilising grouped LEDs with $g=1,2, \cdots, G$ LED arrays used as the transmitters. One method of implementing O-OFDM is to load quadrature amplitude modulated (QAM) complex data symbols onto IFFT subcarriers. In the illustrated system, these subcarriers are partitioned such that the $g^{\text {th }}$ LED array transmits subset of the subcarriers. This partitioning is done with the use of $G$ filtering blocks within the system [9]. Thus, the same signal modulates the intensities of LED arrays in the $g^{\text {th }}$ group with each group having a separate driver.

Let $X_{\mathrm{d}}$ be the data stream intended for transmission. Data sequence $X_{\mathrm{d}}$ is converted from serial to parallel and mapped onto QAM symbols. The complex QAM data symbols form a frame $X_{\mathrm{s}}^{u}(i), \quad i=1,2, \ldots, n_{\mathrm{s}}, u=1,2, \ldots, U$, with $n_{s}$ being the number of active data-carrying subcarriers while $U$ represents the O-OFDM symbols in the frame. The procedure is followed by generation of random sequence of pilot signal $X_{p}(i), \quad i=1,2, \ldots, n_{\mathrm{s}}$. Signal $\mathbf{X}_{p}$ used in rotating the phase of the $\mathbf{X}_{\mathrm{s}}^{u}$ data symbols. Thus, the frame size changes from $U$ to $U+1$ due to the concatenation of the pilot signal. The phase rotation 
procedure can be defined in (11) as:

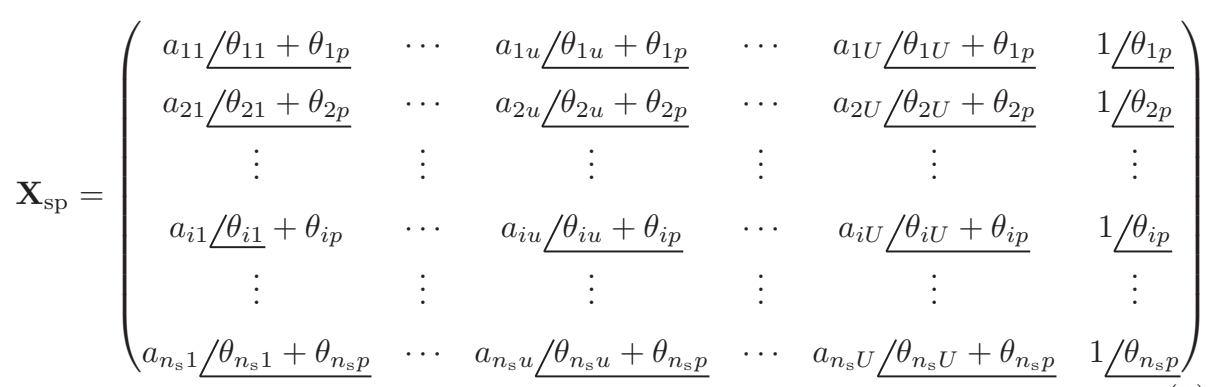

where $a_{i u}$ and $\theta_{i u}$ are the QAM constellation amplitude and phase of the $i^{t h}$ subcarrier and $u^{t h}$ O-OFDM symbol respectively. Also, $\theta_{i \mathrm{p}}$ represents the phase of the $i^{\text {th }}$ pilot symbol which is either 0 or $\pi$. To ensure real-valued signal which is a requirement for intensity modulation and direction detection (IM/DD) system [4], Hermitian symmetry is imposed on the PA O-OFDM frame $\mathbf{X}_{\mathrm{sp}}$. The number of subcarriers increases from $n_{\mathrm{s}}$ to $N=2\left(1+n_{\mathrm{s}}\right)$ as a result of the Hermitian symmetry operation. Thereafter, the PA O-OFDM frame $X^{u}(i), \quad i=0,1,2, \ldots, N-1, u=1,2, \ldots, U+1$ is input to the $G$ filtering blocks.

The filter vector, $\mathbf{F}_{g}$, is given as:

$$
\mathbf{F}_{g}=\left[0, \hat{\mathbf{F}}_{g}(i), 0, \hat{\mathbf{F}}_{g}^{*}(N-i)\right]^{\mathrm{T}} \quad i=1,2, \cdots, n_{s}
$$

where $[\cdot]^{\mathrm{T}}$ denotes transpose of $[\cdot], \mathbf{F}^{*}(\cdot)$ denotes complex conjugate of $\mathbf{F}(\cdot)$,

$$
\hat{\mathbf{F}}_{g}(i)= \begin{cases}1 & i=(g-1) n_{g}+1, \cdots, g n_{g} \\ 0 & \text { otherwise }\end{cases}
$$

for $i=1, \cdots, n_{g}$, and $n_{g}=n_{s} / G$ represents the number of active data-carrying subcarriers per group. Hermitian symmetry is equally ensured on the filter vectors to make sure their output maintains the Hermitian symmetric property required in O-OFDM system. The filters narrow down the band of the PA O-OFDM frame such that its output, $\mathbf{X}_{g}$, represents a distinct subset of the original frame. Thus, the filtering operation reduces the number of active subcarriers that can add up coherently to form high peaks. Filter output $\mathbf{F}_{g}$ is loaded on the $g^{\text {th }}$ IFFT to obtain time domain signal $x_{g}(n)$. 
PAPR of an oversampled discrete time domain O-OFDM signal has been shown to accurately approximate that of a continuous time domain signal [17]. Thus, the PAPR of the continuous time domain signal, $x_{g}(t)$ is approximated by oversampling the PA O-OFDM frame in the frequency domain [12]. The oversampled time domain PA O-OFDM signal, $x_{g}(n)$, is the IFFT of $\mathbf{X}_{g}$. Thereafter, the PAPR of oversampled $x_{g}(n)$ defined as the ratio of the maximum instantaneous power of $x_{g}(n)$ to the average power of $x_{g}(n)$ is evaluated over the PA O-OFDM frame as [12]:

$$
\operatorname{PAPR}_{P_{g}}=\underset{1 \leq p \leq P}{\arg \min }\left[\frac{\max _{0 \leq n \leq(U+1)(N L-1)}\left|x_{g}(n)\right|^{2}}{E\left[\left|x_{g}(n)^{2}\right|\right]}\right],
$$

where $E[\cdot]$ is statistical expectation.

Signal $x_{g}(n)$ with the minimum PAPR per group is obtained by performing $P$ iterations of the data symbol phase rotation with different randomly generated pilot symbol phase sequence. This phase rotation changes the statistical characteristics of the data symbols within the frame in order to reduce the probability of coherent addition of subcarriers that can produce high peaks. Thus, oversampled $x_{g}(n)$ that gives the least PAPR after $P$ iterations is selected to modulate the intensities of LED arrays in the $g^{\text {th }}$ group.

Appropriate DC bias, $\mathcal{B}_{g}$ is added to real-valued bipolar signal $x_{g}(n)$ to obtain unipolar signal, $x_{\mathrm{dc}_{g}}(n)$, needed to drive the $g^{\text {th }}$ optical modulator. It is assumed that all the $\mathcal{D}$ LEDs have the same orientation and are placed such that they have identical gains and similar delays at the receiver [9, 18]. Thus, the system relies on spatial summation of the simultaneously transmitted group signals from the $G$ LED groups during propagation in space. Hence, it is possible to utilise the photodetector of a PA O-OFDM receiver [4] to detect the optical signal at the receiver.

The received signal, $y_{r}(n)$, is proportional to the received power with additive white Gaussian noise (AWGN), $w(n)$, that is:

$$
y_{r}(n)=\frac{\mathcal{D}}{G} \sum_{g=1}^{G} x_{\mathrm{dc}_{g}}(n)+w(n),
$$


where $x_{\mathrm{dc}_{g}}(n)=x_{g}(n)+\mathcal{B}_{g}$. The process of pilot phase estimation using maximum likelihood technique and data symbol recovery at the receiver is similar to the one described in [13].

\section{Analysis of PAPR Distributions of Pilot-assisted O-OFDM System with Grouped LEDs}

In this section, we present the distributions of PAPR of the PA O-OFDM signal utilising grouped LEDs with the assumption that $x_{g}(n)$ follows Gaussian distribution, which is true when $N \geq 64[19]$. In practice, there are two categories of subcarriers in OFDM systems: the active and inactive subcarriers. The data and/or pilot symbols form the active subcarriers, $N_{\text {active }}$, while the null subcarriers represent the inactive subcarriers, $N_{\text {inactive }}$. The transmission power is allocated to active subcarriers in order to have a power efficient system [16]. According to the property of Hermitian symmetry in basic O-OFDM, $X^{u}(0)=X^{u}(N / 2)=0$, making the number of inactive subcarriers relatively small compared to the total number of subcarriers. Hence, it is possible to assume that the total available transmission power is allocated to all $N$ subcarriers. However, this assumption is not applicable for O-OFDM system using grouped LEDs.

Filter output $X_{g}^{u}(i)$ is formed by filtering the original PA O-OFDM frame $X^{u}(i)$ through $\mathbf{F}_{g}$ as in (6):

$$
X_{g}^{u}(i)=\mathbf{F}_{g}(i) X^{u}(i), i=0,1,2, \cdots, N-1 ; u=1,2, \cdots, U .
$$

Assuming equal division of subcarriers among the groups, the Hermitian symmetric filter vector narrows down the number of active subcarriers per group from $2 n_{s}$ to $2 n_{s} / G$. The analytical results established so far on PAPR distributions in discrete multitone (DMT) signal [19] and PA O-OFDM without grouped LEDs 20] are obtained based on the assumption that all subcarriers are fully used as active ones. Thus, it is very important to accurately identify the PAPR distribution in O-OFDM systems where the number of inactive subcarriers are large relative to the total subcarriers. 
Assuming that $N_{\text {active }}$ is large enough, it then follows that the instantaneous power of the $x_{g}(n)$ samples is also Gaussianly distributed [19]. In addition, we assume that active subcarriers have equal power allocation, such that, the power allocated to the $i^{t h}$ subcarrier, $\epsilon_{i}=E\left[\left|X_{g}^{u}(i)\right|^{2}\right] \approx \rho E\left[\left|x_{g}(n)\right|^{2}\right]$, where $\rho \leq$ $N / N_{\text {inactive }}$ for a maximum transmit power constraint. Using these assumptions and the extreme value theory [16, 19], we obtain the cumulative distribution function (CDF) of a single basic O-OFDM symbol in grouped LEDs system as:

$$
F_{Y}(y)=\exp \left[-\sqrt{\frac{4 \rho}{3 N}\left(2 n_{g}^{3}+3 n_{g}^{2}+n_{g}\right)} \mathrm{e}^{-y / 2}\right],
$$

where $F_{Y}(\cdot)$ is the CDF, $N=N_{\text {active }}+N_{\text {inactive }}=2\left(n_{s}+1\right), n_{g}=n_{s} / G$ and $N_{\text {active }}=2\left(n_{s} / G\right)=2 n_{g}$. The derivation of (7) can be extended to cases where active subcarriers have unequal power allocation. Thereafter, we obtain the distribution of PAPR of a single PA O-OFDM frame with the fact that the $U+1$ symbols in the frame have at least $U+1$ distinct maximum peaks. Thus, we use the maximum order statistics [20] and (7) to derive the CDF of PAPR for a PA O-OFDM frame as:

$$
F_{Y_{\left(U_{p}\right)}}(y)=\exp \left[-U_{p} \sqrt{\frac{4 \rho}{3 N}\left(2 n_{g}^{3}+3 n_{g}^{2}+n_{g}\right)} \mathrm{e}^{-y / 2}\right],
$$

where $U_{p}=U+1$. Furthermore, the $P$ iterations of pilot symbol phase rotation with data symbols result in $P$ distinct PA O-OFDM frames. Hence, we use the minimum order statistics [20] and (8) to analyse the PA O-OFDM frame that gives the least PAPR. Thus, we obtain the CCDF of the PA O-OFDM frame with the least PAPR as:

$$
\mathrm{P}_{\mathrm{c}_{\mathrm{g}}}(\mathrm{y})=\left(1-\mathrm{e}^{-U_{p} \sqrt{\frac{4 \rho}{3 N}\left(2 n_{g}^{3}+3 n_{g}^{2}+n_{g}\right)} \mathrm{e}^{-y / 2}}\right)^{P} .
$$

$\mathrm{P}_{\mathrm{c}_{g}}$ is the probability of the PAPR of $x_{g}(n)$ exceeding $y$.

\section{Results and Discussion}

In this section, we conduct computer simulations to validate the proposed analytical CCDF of PAPR in PA O-OFDM systems utilising grouped LEDs. In 
the computer simulations, an oversampling factor of $L=4$ is used in order to obtain approximate PAPR of a continuous time O-OFDM signal and constellation size of 4-QAM is utilised. In this work, perfect synchronization is assumed at the receiving end.

Figure 2 shows the CCDF plot of PAPR for basic and PA O-OFDM system using $P=5, U_{p}=6, G=2, n_{g}=48$ and $N=194$. The accuracy of the derived expression for CCDF of PAPR in (9) is verified by comparison with simulations and presented in this figure. Simulation results show a near-perfect agreement with those obtained using (9) with a gap of about $0.1 \mathrm{~dB}$ due to approximate Gaussianity.

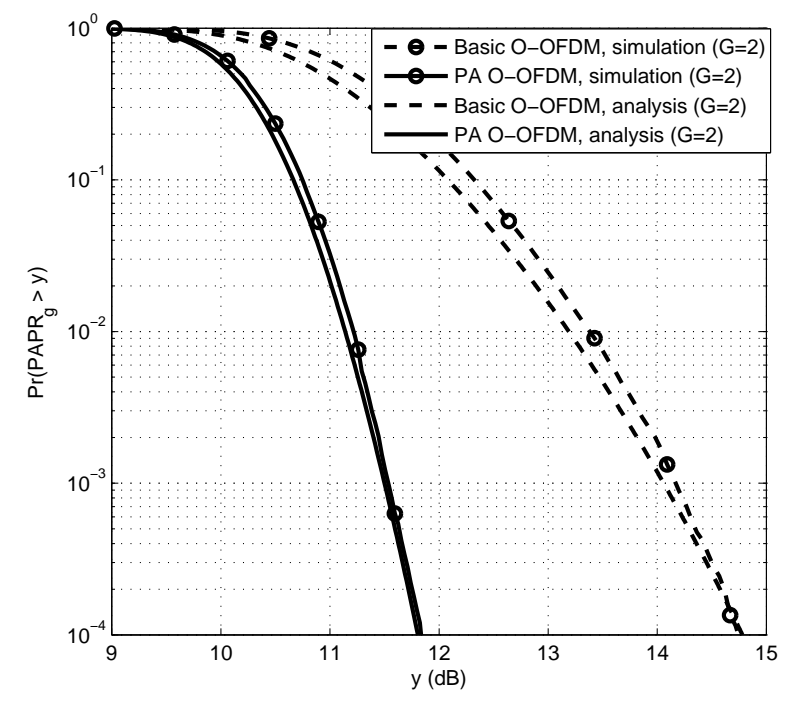

Figure 2: CCDF of PAPR plot for basic and PA O-OFDM signal using $U_{p}=6, P=5, G=2$, $n_{g}=48, \mathcal{D}=96$ and $N=194$.

The threshold PAPR can be obtained from (9) as:

$$
y^{(P)}(\mathrm{dB})=10 \log _{10}\left[-2 \ln \left(-\frac{\ln \left(1-\mathrm{P}_{\mathrm{cg}_{\mathrm{g}}}{ }^{1 / P}\right)}{U_{p} \sqrt{\frac{4 \rho\left(2 n_{g}^{3}+3 n_{g}^{2}+n_{g}\right)}{3 N}}}\right)\right] .
$$

For specific CCDF, (10) gives an idea of $G$ required to achieve a desirable PAPR when $P, N$ and $U_{p}$ are known. Thus, Figure 3 shows a plot of threshold PAPR 
against $G$ for basic and PA O-OFDM with $P=5, N=194$ and $\mathrm{P}_{\mathrm{c}_{\mathrm{g}}}=10^{-3}$. The plot reflects the capability of the PA technique to reduce the number of LED drivers required in O-OFDM system utilising grouped LEDs. The advantage of using multiple LED groups is shown for both basic and PA O-OFDM system because there is decrease in threshold PAPR as $G$ increases. More importantly, comparing the difference in $y$ at every $G$ shows the PAPR reduction capability of the PA technique. For instance, the PAPR reduction gain with the PA technique when $G=3$ is about $2.7 \mathrm{~dB}$. Furthermore, it can be observed that $G=8$ in the basic O-OFDM can only reduce the PAPR to about $13.6 \mathrm{~dB}$ whereas the PA O-OFDM with just $G=2$ is capable of reducing the PAPR to approximately $11.5 \mathrm{~dB}$. This implies that the PA technique can be implemented to limit the hardware complexity arising from use of several LED drivers in the system while having a signal that is less susceptible to clipping due to the limited dynamic range of LEDs.

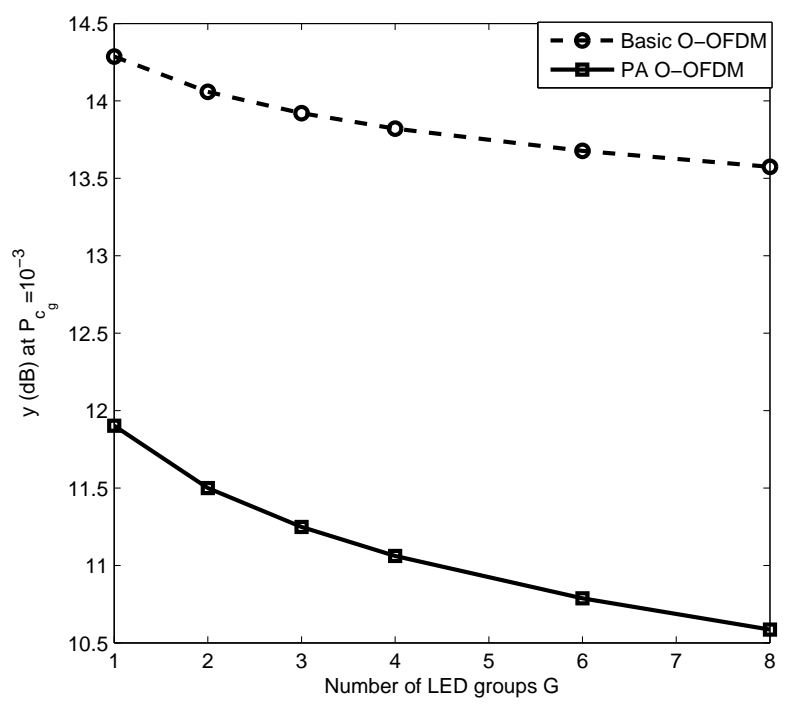

Figure 3: Analytical plot of threshold PAPR against different values of $G$ with $\mathrm{P}_{\mathrm{c}_{\mathrm{g}}}$ of $10^{-3}$ for basic and PA O-OFDM using $P=5, U_{p}=6, \mathcal{D}=96$ and $N=194$.

In Figure团 we compare the error performance of basic and PA system with 
and without grouped LEDs to investigate the effect of clipping induced noise on the O-OFDM signal with high peak values. The normalised upper, $\mathcal{C}_{c_{u}}$, and lower clipping levels, $\mathcal{C}_{c_{l}}$ are defined as: $\mathcal{C}_{c_{u}}=c_{u} \sigma_{x}$ and $\mathcal{C}_{c_{l}}=-c_{l} \sigma_{x}$, where $c_{u}$ and $c_{l}$ are unitless coefficients that determine the severity of clipping experienced by the signal. For the purpose of illustration, the levels are set to $c_{u}=c_{l}=1$ and $\sigma_{x}=250 \mathrm{~mA}$. Other parameters used are $\mathcal{B}_{g}=250 \mathrm{~mA}$ for system with $G=1$ while $\mathcal{B}_{g}=40 \mathrm{~mA}$ for system with $G=3$. As expected, the O-OFDM signals with $G=1$ are more susceptible to clipping than those with $G=3$. However, less clipping distortion is observed in PA O-OFDM with $G=1$ due to the peak reduction capability of the PA technique but the basic O-OFDM signal with $G=1$ has error floor at about bit error rate (BER) of $2 \times 10^{-4}$. Furthermore, the comparison between the basic and PA O-OFDM signal with $G=3$ also shows that the PA O-OFDM requires less optical SNR to achieve target BER than the basic O-OFDM with grouped LEDs.

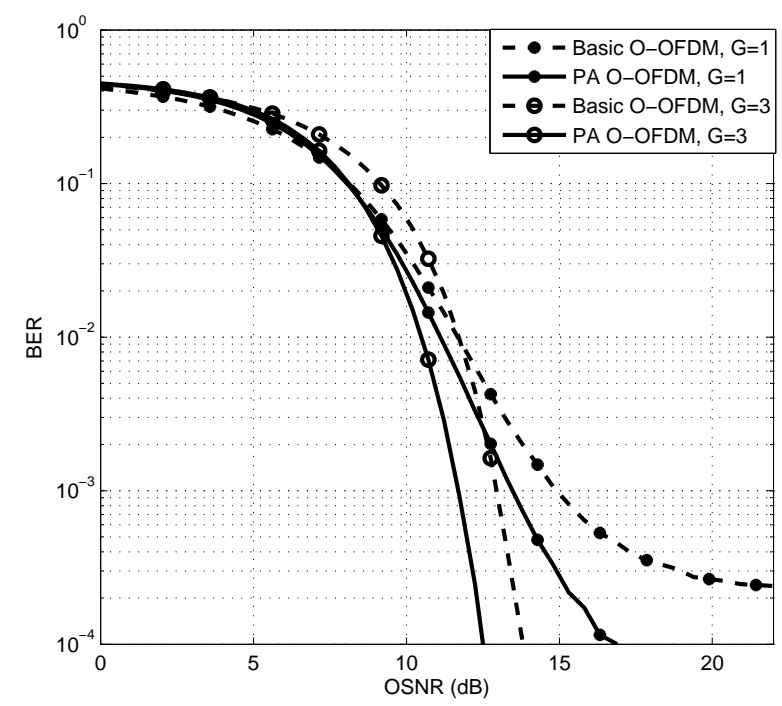

Figure 4: Plot showing the BER performance of basic and PA O-OFDM system with and without grouped LEDs using $U_{p}=6, P=5, G=\{1,3\}, \mathcal{D}=96, N=194$ and 4-QAM. 


\section{Conclusion}

In this paper, we have presented the quantitative analysis of PAPR distributions of the PA technique in O-OFDM utilising grouped LEDs. The PA technique gives further PAPR reduction while minimising the required number of transmit chains in the system. When compared with simulation results, the theoretical results obtained by applying order statistics show nearly-perfect match. The use of PA technique in spatial domain O-OFDM with grouped LEDs provides opportunity for a system design with less hardware complexity, but at the expense of increased computational complexity at the transmitter. Hence, this theoretical study provides a fast and efficient means of determining the trade-off between the PAPR reduction gain required, computational complexity and hardware complexity of the system.

\section{Acknowledgements}

Portions of this work were presented at the $10^{\text {th }}$ IEEE/IET International Symposium on Communication Systems, Networks and Digital Signal Processing (CSNDSP) and IEEE International Conference on Communications (ICC) workshop in 2016 .

\section{References}

[1] Cisco Visual Networking Index, Global mobile data traffic forecast, 20112016 (2012).

[2] Z. Ghassemlooy, W. Popoola, S. Rajbhandari, Optical Wireless Communications: System and Channel Modelling with MATLAB, CRC Press, Boca Raton, USA, 2012.

[3] H. Chun, S. Rajbhandari, G. Faulkner, D. Tsonev, E. Xie, J. McKendry, E. Gu, M. Dawson, D. C. O'Brien, H. Haas, LED based Wavelength Division Multiplexed 10 Gb/s Visible Light Communications, Journal of Lightwave Technology 34 (13) (2016) 3047-3052. 
[4] W. O. Popoola, Z. Ghassemlooy, B. G. Stewart, Pilot-Assisted PAPR Reduction Technique for Optical OFDM Communication Systems, Journal of Lightwave Technology 32 (7) (2014) 1374-1382.

[5] H. Elgala, R. Mesleh, H. Haas, Non-linearity Effects and Predistortion in Optical OFDM Wireless Transmission Using LEDs, International Journal of Ultra Wideband Communications and Systems (IJUWBCS) 1 (2) (2009) $143-150$.

[6] F.-n. Chen, Z.-p. Wang, A post-coding scheme for peak-to-average power ratio reduction in intensity modulated optical OFDM systems, Optoelectronics Letters 10 (2014) 295-298.

[7] T. Zhang, Z. Ghassemlooy, C. Ma, S. Guo, PAPR reduction scheme for ACO-OFDM based visible light communication systems, Optics Communications 383 (2017) 75-80.

[8] H. Dong, H. Zhang, k. Lang, B. Yu, M. Yao, OFDM Visible Light Communication Transmitter Based on LED Array, Chinese Optics Letters 12 (5) (2014) 052 301-1-4.

[9] M. Mossaad, S. Hranilovic, L. Lampe, Visible Light Communications Using OFDM and Multiple LEDs, IEEE Transactions on Communications 63 (11) (2015) 4304-4313.

[10] B. Yu, H. Zhang, L. Wei, J. Song, Subcarrier Grouping OFDM for VisibleLight Communication Systems, IEEE Photonics Journal 7 (5) (2015) 1-12.

[11] R. Lenk, C. Lenk, Practical Lighting Design with LEDs, Hoboken, NJ, USA: Wiley, 2011.

[12] F. B. Ogunkoya, W. O. Popoola, S. Sinanović, Pilot-assisted PAPR Reduction Technique for O-OFDM Using Multiple LEDs in VLC Systems, in: IEEE International Conference on Communications (ICC'16) Workshop, Kuala Lumpur, Malaysia, 2016, pp. 1-6. 
[13] F. Ogunkoya, W. Popoola, S. Sinanović, PAPR Reduction in Optical OFDM with Grouped LEDs, in: 10th IEEE/IET International Symposium on Communication Systems, Networks and Digital Signal Processing (CSNDSP), Prague, Czech Republic, 2016, pp. 1-6.

[14] F. B. Offiong, S. Sinanovic, W. Popoola, On PAPR Reduction in PilotAssisted Optical OFDM Communication Systems, IEEE Access 5 (2017) $8916-8929$.

[15] H.-C. Yang, M.-S. Alouini, Order statistics in wireless communications: diversity, adaptation, and scheduling in MIMO and OFDM systems, Cambridge University Press, 2011.

[16] T. Jiang, M. Guizani, H.-H. Chen, W. Xiang, Y. Wu, Derivation of PAPR distribution for OFDM wireless systems based on extreme value theory, IEEE Transactions on Wireless Communications 7 (4) (2008) 1298-1305.

[17] K. Wong, M.-O. Pun, H. Poor, The Continuous-Time Peak-to-Average Power Ratio of OFDM Signals Using Complex Modulation Schemes, IEEE Transactions on Communications 56 (9) (2008) 1390-1393.

[18] T. Fath, C. Heller, H. Haas, Optical wireless transmitter employing discrete power level stepping, Journal of Lightwave Technology 31 (11) (2013) 17341743.

[19] H. Yu, M. Chen, G. Wei, Distribution of PAR in DMT systems, Electronics Letters 39 (10) (2003) 799-801.

[20] F. Ogunkoya, W. Popoola, A. Shahrabi, S. Sinanovic, Performance Evaluation of Pilot-assisted PAPR Reduction Technique in Optical OFDM Systems, IEEE Photonics Technology Letters 27 (10) (2015) 1088-1091. 